\title{
EL IMPACTO DE LA INNOVACIÓN EN LA PRODUCCIÓN DE MATERIALES PARA LA EDUCACIÓN A DISTANCIA
}

\author{
Luis Fernando Díaz ${ }^{1}$
}

\begin{abstract}
Resumen
Este artículo presenta la experiencia obtenida durante los últimos años en la Dirección de Producción de Materiales de la Universidad Estatal a Distancia (UNED), en especial en el área de producción de material multimedial (PEM). El objetivo es estudiar el impacto de la innovación tecnológica y otros fenómenos análogos en la producción de materiales didácticos en la UNED. Se sigue el método previsto en un proyecto que ha sido desarrollado ex profeso: un observatorio académico que se interesa en el impacto de la innovación tecnológica sobre la educación a distancia. En el texto se analizan diez categorías en el contexto de tarea que impactan de manera principal; dentro de ellas destacan, por su renovada importancia las siguientes: la creciente importancia de la comunicación icónica y gráfica, el potencial de digitalización de "toda" la información, la nueva filosofía del diseño y la desaparición casi absoluta de barreras tecnológicas a la comunicación.
\end{abstract}

\section{Palabras clave}

- Sociedad de la información • Sociedad del conocimiento • Comunicación icónica y gráfica -Digitalización de la información •Barreras a la comunicación •Filosofía del diseño •Paquete tecnológico •Derecho a la información • Programación de computadores •Estrategias de mediación y evaluación • Simuladores

\begin{abstract}
This article presents the experience gained during recent years in the Dirección de Producción de Materiales of the Universidad Estatal a Distancia (UNED), particularly in the area of multimedia production (PEM). The aim is to study the impact of technological innovation and other similar phenomena in the production of educational materials in the UNED. It is followed the method provided by a project that has been developed in PEM: an academic observatory interested in the impact of technological innovation on distance education. The text discusses ten categories making major impact and with renewed importance in the task context, as follows: the growing importance of iconic and graphic communication, the feasibility of digitization of any information, the new philosophy of design and the nearly total disappearance of technological barriers to communication.
\end{abstract}

\footnotetext{
${ }^{11}$ Profesor universitario. Editor y autor académico de la Universidad Estatal a Distancia. 1fdiaz@uned.ac.cr/
} 


\section{Keywords}

-Information society $\bullet$ Knowledge society $\bullet$ Iconic and graphical communication $\bullet$ Digitalization of the information $\bullet$ Communication barriers $\bullet$ Design philosophy $\bullet$ Technological packages -Rights to information

A lo largo de los últimos años, en la Dirección de Producción de Materiales Didácticos (DPM) de la Universidad Estatal a Distancia, especialmente en el Programa de Producción Multimedial (PEM), se ha organizado un proyecto permanente de investigación, denominado OBSERVATORIO, sobre la forma en que las innovaciones tecnológicas inciden en la producción de textos, multimedios y otros productos destinados al uso por la Universidad y los estudiantes como medio para el aprendizaje.

El proyecto ha consistido en la disposición de un grupo de trabajo con una división adecuada de tareas, que permite observar y registrar sistemáticamente los cambios y los eventos que caracterizan y modifican el estado del arte, entendido como el nivel más alto de desarrollo -o vanguardia- en un momento dado en un campo del conocimiento. En nuestro caso, se trata del impacto de la innovación tecnológica y la movilidad social sobre la enseñanza a distancia.

El medio en el cual se genera y se produce la incorporación de innovaciones en la educación a distancia puede ser estudiado desde numerosas perspectivas, y utilizando instrumentos de diversas disciplinas profesionales. En este caso, en razón de la importancia del proceso social de difusión/adopción de innovaciones, el modelo que se utiliza es propio del campo de la comunicación social. La premisa fundamental es que la forma en que se intercambia conocimiento en la sociedad se ha modificado significativamente en un periodo corto y relevante de tiempo, al grado que la cualidad más importante en esa nueva sociedad es precisamente el intercambio de información. De idéntica forma, además de la transformación del contenido de la comunicación, se han modificado los medios y las estructuras utilizadas para el intercambio.

En este OBSERVATORIO el enfoque que se ha empleado es esencialmente cualitativo. Se ha elaborado un mapa de variables y relaciones que, de acuerdo con el juicio de expertos, son determinantes de la innovación en medios y materiales. En el contexto relevante o "de tarea", para efectos de este trabajo, se distinguen diez grandes categorías que corresponden a elementos diferentes (a veces momentos o ámbitos) en que se manifiestan las variables que cambian de manera más significativa.

Las categorías que se reconocen -y que se comentan en las páginas siguientes- son:

- El surgimiento de la sociedad de la información o sociedad del conocimiento.

- La información como bien y como objeto de derechos individuales.

- Preeminencia de la comunicación icónica y gráfica.

- Viabilidad de la digitalización de "toda" la información.

- Desproporción en la innovación en software vs. hardware.

- Aceleración en la velocidad de desarrollo de software.

- Desaparición de barreras tecnológicas importantes a la comunicación. 
- La nueva filosofía del diseño.

- Desaparición de las diferencias entre medios y materiales.

- Otros cambios que configuran un nuevo contexto universitario (nuevo perfil de la relación entre la universidad y la sociedad).

De esas categorías, cuál es el cambio o condición en el medio que propicia e induce la innovación es difícil de determinar. Antes se usaba decir que los cambios tecnológicos eran el resultado de modificaciones en la cultura; hoy, en numerosas ocasiones son las transformaciones tecnológicas las que arrastran en su dinámica a las nuevas formas culturales y sociales. "Hay una percepción desconcertante que ahora la tecnología tira de la cultura" (Johnson, 2006, p.23). En realidad se presenta una especie de ciclicidad en que ciertos cambios en técnicas, procedimientos o instrumentos, parecen empujar modificaciones en la aceptación y en los usos que se le brindan a esos medios, incluyendo su reproducción. Lo que sucede tiene que ver con el hecho de que en la práctica las tecnologías no ocurren en el vacío, sino que parte de ella son las condiciones de mercadeo y difusión, así como las instrucciones para la operación y los programas de capacitación. En suma: el llamado paquete tecnológico.

A continuación se presenta, para cada una de las categorías indicadas, la forma en que se percibe desde el OBSERVATORIO así como algunas de las decisiones principales que resultan en la producción de materiales, como respuesta a su influencia.

\section{El surgimiento de la sociedad de la información o sociedad del conocimiento}

El sistema social actual, que indistintamente se ha dado en llamar sociedad de la información o sociedad del conocimiento, no tiene en realidad similitud con ninguna forma anterior, y el uso de conceptos y nombres de otras épocas y de otras formas de organización humana es meramente un recurso analógico y heurístico para caracterizar e identificar esa nueva formación social. Esta categoría general se refiere a la configuración del medio social actual como un arreglo nuevo y muy distinto de todas las formas sociales anteriores.

El doble nombre no es un accidente ni resulta de incertidumbre sobre la naturaleza del fenómeno. Reconoce más bien la importancia del análisis de los dos aspectos en forma conjunta, en el sentido de que interesa el valor de la información en razón del conocimiento como finalidad intermedia.

Algunas consecuencias o peculiaridades de esta sociedad se tratan como parte del esquema central en las categorías que se presentan más adelante. Sobre otras, por su mayor generalidad y, al mismo tiempo, por su relación directa con los procesos de aprendizaje, se comparten de seguido las ideas aportadas por Bárbara Fabré:

"En la sociedad del conocimiento...con un uso intensivo de la información, en la que el ciudadano interactúa con personas y máquinas en un constante intercambio de datos e información, la alfabetización tradicional, las habilidades de lecto-escritura, que constituyen la base de los sistemas educativos primarios, no es suficiente. A estas habilidades hay que añadir nuevas habilidades informacionales, como la consistente (sic) en saber navegar por fuentes "infinitas" de información, saber utilizar los sistemas de información, saber discriminar la calidad de la fuente, saber determinar la fiabilidad de la fuente, saber dominar la sobrecarga informacional o "intoxicación", saber aplicar la información a problemas 
reales, saber comunicar la información encontrada a otros, y, más que otras cosas, saber utilizar el tiempo, el verdadero recurso escaso en la sociedad del conocimiento, para aprender constantemente.

Pero quizás más importante que enseñar será posiblemente cómo enseñar. En un mundo repleto de información, que llega por múltiples canales, mantener la atención del "estudiante" será muy difícil. Será preciso desarrollar nuevos métodos de enseñanza fundamentados en la idea de estímulo continuo. Por una parte, atraer la atención de quien debe aprender, sólo podrá conseguirse mediante la conversión del proceso de aprendizaje en otro de descubrimiento, de implicación, de satisfacción de la curiosidad con un alto componente de diversión. Y satisfacer a quién quiere aprender, implicará que se responde de forma personalizada a sus necesidades y que se compensa adecuadamente el esfuerzo que se invierte en el aprendizaje.” (Fabré, 2006, s.n.)

En una sociedad como ésta el cambio es una condición constante e inherente al sistema. De manera que la aparición y la adopción de innovaciones no son eventos extraños, sino, más bien, parte de la dinámica habitual. Hoy, aunque nos sorprenda, lo verdaderamente raro es la ausencia de cambios: la estabilidad en los sistemas - de cualquier tipo- solo se sostiene por diseño, y el plan -todo plan- tiene que contemplar la caducidad de las soluciones y la prevención de la obsolescencia.

\section{La información como bien y como un objeto de derechos individuales}

En esta nueva configuración social, la información es tanto un bien como un objeto de derechos individuales. Por eso, la caracterización del contexto debe considerar las condiciones de administración y uso de la información que contemplen las múltiples dimensiones en que los individuos se relacionan con ellas. Algunos de los aspectos en esta área se refieren a factores tales como "mercados" de información, precios y demanda, su naturaleza como bien libre, monopolios y otros elementos similares.

Por otro lado, el derecho a la información - por ser multidimensional- configura un campo vasto, diverso y disperso. Se trata en realidad de varios tipos de derechos: derechos humanos, derechos morales, derechos pecuniarios y sistemas de propiedad, e incluye, como dimensiones principales, los siguientes:

- el derecho a disponer libremente de la información producida por uno mismo.

- el derecho a la confidencialidad de la información sobre uno.

- el derecho a acceder a toda la información sobre uno y a toda la información considerada pública, o que pueda afectar el disfrute de los otros derechos.

La innovación en el campo de la producción de materiales educativos encuentra límites reales, aunque a veces contradictorios, en todos esos derechos. Manifestaciones concretas de problemas se dan en relación con diversos grados de reconocimiento de créditos y méritos académicos así como derechos de autor y también a las violaciones, encabezadas por el plagio, la fabricación y la falsificación (National Academy of Sciences -USA- ,1993). Un aspecto central en la adopción de innovaciones tecnológicas es el que se relaciona con la propiedad del software por utilizar. Esto 
incluye condiciones como el pago de licencias, la recurrencia y el costo de las actualizaciones, todo confrontado con el uso de software libre.

En cuanto al uso de software libre, se distinguen como características importantes: la libertad de uso, la libertad de adaptación y el acceso al código fuente de los programas, la libertad de compartir y la libertad de mejorar el programa y de compartir esas mejoras con terceros. Otros temas que deben considerarse es que libre y semilibre son condiciones que no siempre conllevan la gratuidad en todas las dimensiones del uso.

Hoy, en la Dirección de Producción de Materiales de la UNED se enfrenta esta problemática desde diversas perspectivas sobre numerosos desarrollos, entre los cuales se incluyen investigación y desarrollo de plataformas alternativas para la gestión de cursos en línea. Los esfuerzos principales se centran en el fortalecimiento de las capacidades para el uso de WebCT, que es el sistema bajo contrato y de la opción de software libre usada localmente por más de siete años (Microcampus). Pero ahora se está afrontando el uso de Moodle como soporte de comunidades de aprendizaje basadas en mayor participación de los estudiantes. Al mismo tiempo se está explorando el utilizar Sakai, que se ha venido convirtiendo en un recurso popular entre las universidades más reconocidas. Sakai se utiliza en la Universidad de Guadalajara, que tiene un convenio con la UNED, para el desarrollo de objetos de aprendizaje y de soporte de materiales para cursos en línea.

De la misma forma, se está estudiando el potencial de Creative commons en un sistema de educación a distancia como el nuestro. Creative commons de Massachusetts es una corporación sin fines de lucro que se encarga de proveer herramientas sin costo a los autores, artistas, científicos y educadores para que identifiquen y distribuyan su trabajo y sus obras con los grados de libertad que deseen.

\section{Preeminencia de la comunicación icónica y gráfica}

La simplicidad, la economía de representación y el valor metafórico que aportan las imágenes visuales han hecho que en el mundo actual se produzca una clara preferencia por la comunicación gráfica. "Vivimos en una auténtica iconosfera", afirman Villafañe y Mínguez (2002).

Además, las técnicas para desarrollar, producir, animar y reproducir imágenes se han multiplicado. Se cuenta con nuevos materiales (tintas, papeles), nuevos soportes (físicos y digitales, fotosensibles y magnéticos), nuevas técnicas (la macroserigrafía, el "ploteado" y la impresión en gran formato) y nuevos medios (la TV digital, el cable TV). Y se sabe mucho más sobre todos ellos; por ejemplo, se dispone de minucioso conocimiento sobre la semántica visual y la sicología del color. El diseño gráfico, el diseño comercial, el paisajismo urbanístico, la arquitectura, y otros campos afines se han establecido como disciplinas universitarias o técnicas profesionales y laborales. También se cuenta con nuevos conocimientos en campos como la sicología aplicada y las ciencias cognoscitivas que nos revelan la importancia y el significado de la comunicación no verbal, del lenguaje corporal e incluso de sutilezas en el campo de la imagen como, por ejemplo, los “contornos dibujados en el aire” (Villafañe y Mínguez, 2002, p. 51) o el llamado "arte de los entornos" o "envolturas monumentales" de Christo Javacheff. 
Hoy, las características más relevantes incluyen, primero, la omnipresencia del fenómeno de la comunicación gráfica. Dondequiera que miremos, la comunicación -0 , por lo menos, los mensajes- nos invaden. Destaca en segundo lugar la síntesis de los recursos icónicos y los tipográficos (incluso con otros, como los sonoros), convirtiéndose el mensaje en una amalgama integrada de sentido. Finalmente, el mundo de la imagen es móvil, dinámico, adaptable y muy cambiante; gracias al cine y a la ilustración digital animada, la comunicación integra una nueva dimensión dinámica: el tiempo, y con ella, al mismo tiempo, el carácter mutable, fugaz y efímero de las formas.

Obviamente, la comunicación mediante imágenes es un fenómeno social que no es nuevo. La práctica y sus evidencias se encuentran en todas las manifestaciones artísticas y comunicacionales humanas desde la prehistoria. La multiplicidad y la riqueza de los soportes (desde arcilla, piedra, papiro, pergamino, cerámica y madera, hasta monedas y construcciones monumentales como pirámides y obeliscos, pasando por relieves, frescos y lápidas) son prueba del ingenio, la diversidad y la difusión temprana de la comunicación icónica y gráfica.

Sin embargo, hay un cambio en la percepción consciente del valor de esa comunicación que se inicia, posiblemente, con los carteles parisinos a finales del siglo XIX y que se convierte en una mirada introspectiva completa con la creación del Bauhaus en 1919 y, más concretamente, con sus primeras expresiones explicativas en 1923.

En otro orden, en los últimos tiempos se ha venido profundizando en la exploración introspectiva del campo de la comunicación iconográfica, al grado que recientemente se han propuesto diversas expresiones formales del conocimiento, particularmente en lo que se ha empezado a conocer como Teoría General de la Imagen. En algunos ambientes, el conocimiento y el uso de los fundamentos de esa Teoría General de la Imagen son esenciales en los programas de estudios en el campo de la comunicación; en la UNED, su dominio empieza a considerarse una parte indispensable de la producción de materiales didácticos, especialmente los multimedia.

La investigación en el PEM se ha centrado en las posibilidades de poner en práctica, en el diseño de las unidades didácticas, tanto impresas como multimediales, algunas de las categorías expuestas en el modelo de Lidwell et al. (2003). El marco teórico general que se ha venido elaborando incluye, entre otros, los siguientes componentes paradigmáticos:

- El conocimiento como diseño y el diseño como una estructura adaptada para un propósito (Perkins, 1989).

- El valor universal de los principios generales del diseño (Lidwell et al., 2003).

- La relación de los individuos con los objetos de diseño es, además de reflexiva y conductual, también emocional (Norman, 2005).

Este fenómeno se comenta con un mayor grado de detalle en una sección posterior (8. La nueva filosofía del diseño).

\section{Viabilidad de la digitalización de "toda" la información}


Mucho del cambio que impacta a la información, tanto a la que se expone en forma verbal como a la que se ofrece en forma gráfica, radica en las facilidades con que se cuenta hoy para la captura de los datos (o los componentes esenciales fundamentales, como puntos, píxeles o bits).

La digitalización es el proceso mediante el cual la información analógica (fotos, un texto, una composición musical) se convierte en una entidad discreta o en una serie de datos manejable por un computador. En la actualidad, prácticamente no existen restricciones para convertir cualquier tipo de información, mensaje, imagen, e incluso "cuerpos sólidos" en reproducciones económicas pero fieles, que pueden guardarse en computadores o en medios portátiles de almacenamiento (discos, llaves USB, tarjetas de memoria tipo flash cards o memory stick) y transmitirse, por distintos y múltiples conductos de un computador a otro o, incluso, a una diversidad de aparatos receptores y reproductores. Es importante considerar que cualquier representación digital puede mantener la calidad gráfica de su generador analógico.

La digitalización ha hecho posible un lenguaje único para la fotografía, la edición, la televisión y la comunicación en redes. Una consecuencia de gran impacto en el campo de la producción de materiales didácticos es la economía de recursos, pues una sola creación puede utilizarse para la difusión por diversos medios y, en general, puede contarse con extensos bancos de información compartidos. Además, independientemente de la peculiaridad del diseño que exige cada uno de los medios, ciertamente se logran las ventajas para el reforzamiento del aprendizaje que resulta de la repetición coherente del mensaje.

En el campo editorial, la industria entera se ha transformado: todo el sistema de reuniones, discusiones y revisiones se ha sustituido por enlaces y conferencias virtuales, y todas las fases del diseño y la pre-prensa se realizan sin ver copias en papel. En las universidades más grandes y reconocidas se ha utilizado todo este nuevo potencial para la producción de materiales ágiles, dinámicos e intercambiables. Se trata de la investigación ya madura en el campo de los llamados objetos de aprendizaje, que son pequeñas unidades de contenido que pueden ser utilizadas, actualizadas, adaptadas y catalogadas utilizando facilidades propias de las plataformas de aprendizaje en línea. Los propósitos de aprendizaje y toda la planeación y gestión del sistema deben de ser parte del diseño.

En la DPM, las diversas oficinas han iniciado ya proyectos específicos en el campo del diseño de objetos de aprendizaje. Por un lado, la conversión fácil y necesaria del enorme bagaje audiovisual con que se cuenta, en objetos transportables digitalizados se ha venido completando de manera sistemática; y en Videoconferencias y en Aprendizaje en Línea ya se acometen intentos de experimentación con materiales análogos. Ahora la DPM se encuentra abocada al desarrollo de criterios marco para el diseño así como el almacenamiento en repositorios compartidos.

\section{Desproporción en la innovación en software vs. hardware}

Hasta hace muy pocos años, la variable crítica de los sistemas computarizados era la disponibilidad de interfases en los límites de entrada de los datos y de salida de la información. Pero esta es una dificultad que ya ha sido superada y es posible afirmar que prácticamente no existen barreras para capturar información en cualquier forma en que se presente. El futuro en este campo es todavía impredecible, pero se puede aseverar con cierta confianza que los grandes 
retos planteados para el desarrollo de equipos de cómputo han sido superados con la eliminación de las restricciones a tales interfases.

Los otros dos indicadores de rendimiento del hardware, la cabida de memoria y la velocidad de procesamiento, han superado ya por mucho la capacidad que tienen los individuos de usar toda la información acumulada, de manera significativa, en lapsos definidos de tiempo. Esta es una de las consecuencias más relevantes de los fenómenos que estudia la Ley de Moore (1965). La Ley de Moore, actualizada en 1975 propone que el número de transistores en un chip se duplica cada dos años, lo que se sigue cumpliendo hoy. Además de proyectar el número y la complejidad de los chips (número de transistores en un chip), esta Ley sugiere también una tendencia a la disminución de los costos. A medida que los componentes de las computadoras se vuelven exponencialmente más económicos, su oferta es mayor y su presencia más extendida.

Estas características de modernidad y efectividad del hardware han propiciado que buena parte de la demanda y del impulso para el desarrollo se haya centrado, en los últimos años, en aplicaciones, programas, paquetes, plataformas, en general software, entendido como estructuras, reglas y procedimientos y su documentación. "Todas las innovaciones profundas de los últimos quince años provienen del software”, dice Johnson (2006).

Además, la tendencia a fortalecerse que han mostrado los movimientos pro software libre se nutre en forma constante de sí misma, lo que ha facilitado un proceso continuo de crecimiento y mejoramiento. El resultado es que la tendencia al surgimiento de innovaciones en software es significativamente mayor que en hardware.

Sin embargo, esta onda expansiva acarrea algunos efectos negativos. Por ejemplo, como el software libre es, precisamente, libre, algunos usuarios simplemente acceden a él en su forma primitiva y pretenden usarlo durante largos periodos sin perfeccionarlo. El caso es que no existen protocolos de actualización o mejora mediante convenio o contrato con los productores, en especial porque la figura del dueño o custodio no existe. Cuanto más elaborado y complejo es el sistema, como las plataformas de aprendizaje en línea, más susceptible es a este tipo de desgaste u obsolescencia.

En la Dirección de Producción de Materiales de la UNED, se han preparado protocolos para el uso del software libre y, especialmente, el gratuito, con la intención de minimizar los riesgos del uso indiscriminado y acrítico. Las normas de control de calidad en el PEM y el protocolo del PAL para el uso y el diseño de cursos utilizando Moodle son dos ejemplos característicos.

\section{Aceleración en la velocidad de desarrollo de software}

La industria del software es muy dinámica porque prácticamente no existen barreras de entrada, $\mathrm{y}$ la distancia (en recursos monetarios y en inteligencia) para pasar de las ideas a los productos es relativamente corta, en comparación con otros tipos de productos o industrias de tecnología pesada con fuertes tendencias hacia la concentración. La ausencia de barreras de entrada favorece la competencia entre creativos y diseñadores, y se facilita así la innovación.

Una buena parte del software se desarrolla con la intención de solucionar problemas concretos con base en una estructura de pensamiento casi personal o de pequeños grupos. Esa parroquialidad aparente, que parece ser parte de los disparadores de la creatividad en este campo, 
tiende también a fortalecerse en el tanto en que propicia la construcción o identificación de nichos o segmentos de mercado casi cautivos.

Todo esto contribuye a que la velocidad de desarrollo de software sea muy alta. Por otro lado, aunque parezca paradójico, tanto la existencia de software libre, como de sistemas protegidos por la legislación de derechos de autor, propician una mayor velocidad en el desarrollo de aplicaciones, en el primer caso por las adhesiones y contribuciones que genera, en el segundo por el creciente potencial de captación de recursos de inversionistas que se genera.

La adopción de nuevos paquetes con altísima velocidad se ha vuelto una de las características más relevantes del PEM. Sin embargo, es claro que esta tendencia ejerce una considerable presión sobre los programas de capacitación y sobre este mismo OBSERVATORIO.

\section{Desaparición de barreras tecnológicas importantes a la comunicación}

Una de las características más peculiares de la sociedad actual es el fenómeno denominado aldea global, el que hace referencia, entre otras cosas, a que prácticamente no existe evento importante, que tenga lugar en cualquier parte de la tierra, que no pueda ser registrado o visto en tiempo real por los más amplios auditorios humanos.

Los avances en las ciencias de la comunicación, incluyendo mejoras sustanciales en emisores y receptores, así como en medios y materiales para la trasmisión, han hecho desaparecer numerosas barreras, pero en especial las distancias. También se ha superado notablemente la velocidad de comunicación, debido en especial a los nuevos materiales para la trasmisión de datos, como la fibra óptica.

En los campos en que se han dado los mayores cambios se encuentran las conexiones de redes de alta velocidad, con capacidad para multiplicar la cantidad de datos que se pueden enviar a la vez (banda ancha). Un caso relevante son las redes de alta velocidad destinadas a servicios especializados como Internet 2, diseñadas para fines académicos. En nuestro medio, la explotación del potencial de esta red para la educación a distancia está aún pendiente, dado que todavía no ha trascendido las etapas experimentales.

Por otro lado, ya se encuentran en funcionamiento diversas redes inalámbricas, que son sistemas de dispositivos electrónicos que no necesitan cables para establecer la comunicación, sino que utilizan ondas radio magnéticas o rayos infrarrojos. Actualmente se utiliza un conjunto de estándares de transmisión para redes inalámbricas -la llamada WiFi, por Wireless Fidelity-con un alcance de poco más de una centena de metros. Con tecnologías similares y con el apoyo de satélites, en algunos países ya están funcionando comercialmente redes inalámbricas para la trasmisión de televisión digital. También se ha desarrollado un sistema de conexión inalámbrica, similar al WiFi, llamado WiMAX que permite conexiones de hasta 70 Mbits por segundo, en comparación con 54 Mbits por segundo que alcanzan las WiFi. En estas redes, el área de conexión puede cubrir hasta 48 kilómetros de radio, unas 300 veces más que el WiFi. Con una instalación como esta se podría conectar de manera inalámbrica la ciudad de San José completa, incluyendo numerosas poblaciones de Heredia y Alajuela, y, si no fuera por el cerro de Ochomogo, parte del valle de Cartago. 
Todos estos sistemas y recursos, empezando con la que ahora nos parece "vieja" Internet, nos proporcionan acceso irrestricto a innumerables y ricas fuentes de cultura y conocimiento. Dentro de ellas, por su aplicabilidad inmediata en el campo educativo, destacan los museos, los archivos institucionales y estatales, y las bibliotecas virtuales. Algunos de esos recursos son utilizados en la UNED en producciones de multimedios y en aprendizaje en línea. Un ejemplo representativo es un carrusel construido con pinturas seleccionadas del Museo del Prado, bajo autorización expresa, ilustrando un uso típico en la unidad didáctica multimedial Internet llega al aula.

Sin embargo, como se ha señalado, en la mayoría de estas facilidades la UNED tiene todavía pendiente la tarea de investigar y crear aplicaciones. En un futuro próximo, al desarrollarse relaciones de cooperación entre las direcciones de Producción de Materiales y de Tecnologías y Comunicaciones, este es el tipo de progreso que debería fructificar.

\section{La nueva filosofía del diseño}

La forma en que se entiende y se valora el papel del diseño en la sociedad actual es notablemente distinta de la que era predominante a mitad del siglo anterior. Entonces, el énfasis se depositaba en los elementos ambientales y gráficos, especialmente por el uso que de sus conceptos se hacía en disciplinas profesionales como la arquitectura y el diseño gráfico. En campos como el periodismo, la publicidad, la propaganda, el diseño de interiores y la moda, se aprovecharon los recursos y las competencias que provenían de la formación académica, profesional y técnica en diseño. Escenarios como las guerras (especialmente la Segunda Guerra Mundial) y el auge de la televisión comercial en la década de los años 50, abrieron posibilidades para la experimentación con nuevos enfoques y técnicas en el manejo de la imagen, así como en el uso de medios y soportes alternativos. Hacia atrás en la historia, por lapsos de siglos que varían dependiendo de la cultura específica, estos temas eran parte central de la producción de libros y también de tapices (y alfombras), pero difícilmente se proyectaban más allá. Un evento novedoso que ya se ha comentado fue la pintura de carteles a finales del siglo XIX, la que, por cierto, se revisa y se renueva con la publicidad en Norteamérica en los años 20 y con la propaganda de las campañas bélicas (en especial en Alemania y en Gran Bretaña durante la tercera y la cuarta décadas).

Hoy, la definición del diseño, aunque se relaciona con el manejo de imágenes y modelos, se centra más en la racionalidad y la intencionalidad que se encuentran en la base del objeto. Heskett lo define como la capacidad humana para concebir y moldear nuestro entorno, sin basarse en antecedentes presentes en la naturaleza, para atender a nuestras necesidades y dar sentido a nuestra vidas (2002, p.5-7). En forma coherente, aunque no coincidente, Perkins afirma que diseño es cualquier estructura orientada a cumplir un propósito (1985, p.19 y sig.). Perkins realiza además una especie de definición circular que lo lleva a sostener que el conocimiento (relevante) es información y que la información es diseño. Dice: conocimiento es "fragmentos de información como estructuras adaptadas con un propósito" (Ib. 21).

La nueva filosofía del diseño, incluyendo ricas dimensiones epistemológicas y metodológicas, es una de las notables innovaciones en el contexto que ofrece un impacto heurístico y relevante en la producción de materiales didácticos. Las obras de Perkins y de Heskett son ejemplos visibles del clima actual de la discusión, pero existe en realidad un grupo grande de estudiosos 
comprometidos con el tema, dentro de los cuales destacan los numerosos trabajos de Steven Heller y de DK Holland (véase la bibliografía).

Una aplicación concreta en el campo de la educación se encuentra en la producción de materiales didácticos en la Universidad de Guelph, Canadá, que sigue los denominados Principios Universales del Diseño Instruccional, cuyo empleo se procura también en todas las otras actividades de enseñanza y aprendizaje, en especial la tutoría. Otra aplicación interesante, que se encuentra en algunas páginas WWW seleccionadas (ver (Multimedia, 1998) es la metáfora comunicacional, elemento de diseño que sirve como leit motiv y como hilo conductor en la totalidad de un sitio web, contribuye a orientar al usuario en su navegación e incrementa con ello su identificación y lealtad. En el caso de Programa de Producción Multimedial (PEM), la metáfora se ha convertido en un criterio esencial de control de calidad, en una característica obligada del diseño y en un elemento común a toda unidad didáctica que integre materiales producidos para distintos medios. Los buenos sitios WWW, las buenas páginas institucionales y los cursos sobresalientes en Internet cuentan con metáforas poderosas, significativas y de gran valor estético. En conclusión, el uso de la metáfora pedagógica como motor del diseño es una innovación mayor, todavía prometedora, en el campo de la creatividad aplicada a la producción de materiales educativos.

Uno de los aportes más reveladores en este campo es la propuesta de los principios del diseño universal, recogidos en la obra homónima de Lidwell et al. En este libro, que es una especie de manual de la buena comunicación, se proponen "Cien maneras de fomentar el utilitarismo, influir en la percepción, incrementar el atractivo de los objetos, diseñar de forma más acertada y enseñar a través del diseño" (Lidwell et al., 2005, p.3).

Por último, hay que tomar en cuenta los trabajos de Donald A. Norman, en especial su última obra, El diseño emocional, que se ocupa de la relación entre el diseño de las cosas, en general, y sus efectos sobre las personas. Norman afirma:

Investigaciones recientes han demostrado cómo los objetos que nos resultan atractivos funcionan, de hecho, mucho mejor. No nos limitamos a usar un producto, sino que establecemos una relación emocional con él.

En la creación de un producto, el diseñador tiene que considerar muchos factores: la elección del material, el método de fabricación, el modo en que el producto es lanzado al mercado, el coste y la utilidad práctica, y también lo fácil que es utilizarlo, comprenderlo. Pero lo que muchos diseñadores no perciben es que existe también un fuerte componente emocional en el modo en que los productos son diseñados y utilizados. (2004: 18-20).

Algo notable que ha sucedido con muchos de estos libros, cuyas ideas constituyen ya innovación -innovación y fuerza precursora y transformadora en el campo del diseño- es que han sido publicados simultáneamente en muchos idiomas, incluyendo el texto original en inglés y la traducción al español, como si fueran parte de un proyecto y una estrategia comunicacional. Dentro de ellos destacan el de Norman; el de Heskett; El ABC de la Bauhaus y la teoría del diseño, de Lupton y Miller; y el de Lidwell et al. que comentáramos antes: Principios Universales de Diseño.

\section{Desaparición de las diferencias entre medios y materiales}


La unidad de concepto y de uso a que conducen los cambios que se han enumerado en la sección anterior en el campo del diseño y, al mismo tiempo, las facilidades casi invasivas en los procesos de digitalización (ver sección 4), han conducido a una virtual desaparición de las diferencias entre los materiales didácticos y los medios requeridos para su difusión.

Más aún, la integración de todos los elementos en la entrega de la docencia, ya sea personal, ya por conferencia remota o por medio de materiales impresos o digitales, ha llevado a que desaparezcan las diferencias entre el diseño, los medios y los materiales que se usan para trasmitir los contenidos.

En los trabajos que se realizan cotidianamente en la DPM se enfrenta esta problemática como parte fundamental del proceso. Al planear y al escribir un material, las decisiones sobre contenido y sobre la forma en que ese contenido se presenta y se distribuye -i.e. tamaño de los párrafos, tipografías, color, etc.- tienen que hacerse de manera simultánea con la adopción de decisiones sobre el medio de salida. Un texto aparentemente idéntico, adopta características distintivas dependiendo de si se va a imprimir en un formato tradicional, como un libro o folleto, o si se va a distribuir por la red utilizando algún sistema de aprendizaje en línea. Y, en este último caso, deben considerarse las condiciones previstas para la impresión o reproducción personal, que apunta a un formato PDF, o si se va a proponer como texto en un modelo más libre y adecuado para la lectura en pantalla. Por supuesto, cada medio exige cierto grado de especificidad: no es lo mismo el lenguaje de la televisión que el de videoconferencias. Pero, al mismo tiempo, las condiciones de diseño compartidas se incrementan cada vez más en número y en importancia.

El diseño de materiales didácticos, en la práctica en la DPM, tiene que resolver continuamente esta aparente paradoja. Es decir, al mismo tiempo debe generalizar y debe particularizar. Debe generalizar en todo aquello que maximice el uso eficiente de los procesos digitales y la información digitalizada, así como en la medida en que se garantiza de forma apropiada la redundancia que se requiere para asegurar el acceso de todos los estudiantes a la información. Debe particularizar en todo aquello que demanda el uso eficaz de cada medio para la trasmisión inequívoca y rica de los contenidos de aprendizaje.

\section{Nuevo perfil de la relación entre la universidad y la sociedad}

Algunos otros cambios importantes en la sociedad, que forman parte de ese contexto que es determinante para la adopción de innovaciones en educación, incluyen tres muy importantes que configuran un nuevo perfil característico de las relaciones entre la universidad y la sociedad más amplia en la que se inserta. Estos son:

- Los cambios en el mercado laboral.

- La nueva ética universitaria.

- La expansión de la oferta de programas universitarios desde y hacia el exterior.

Cambios en el mercado laboral. Hace apenas unas pocas décadas, al ingresar a realizar estudios universitarios, los jóvenes se encontraban con un conjunto de carreras profesionales altamente estructuradas. Los programas de estudios y los perfiles de entrada y de salida correspondían claramente con una imagen idealizada a partir de las llamadas profesiones liberales. Es llamativo como, en la década de los años sesenta -iapenas ayer!-, las opciones retadoras se encontraban en 
los campos relacionados con educación, filosofía y, tímidamente, en las ciencias sociales, incluyendo periodismo y ciencias políticas. Hoy, en cambio, esas son alternativas de salida estables para los graduados universitarios. Y son otras las carreras que prometen alguna aventura y que se perciben como nuevas, entre las que se incluyen algunas relacionadas con campos de actividad que se han comentado en estas páginas, como las de realizador multimedial o diseñador de materiales para redes de comunicaciones digitales, o las de editor o productor, y muchas similares. Las nuevas profesiones y especialidades se encuentran en todos los campos. Los empleadores ahora tienden a reclutar personal con base en perfiles particulares adaptados a las necesidades del puesto, más que centrados en las competencias previstas en el programa de estudios. Es claro que las universidades y los institutos aprenden y modifican, a veces bastante rápido, las bases de reclutamiento.

Además, el cambio empieza a expandirse, por ejemplo: la Cátedra UNESCO de Educación a Distancia de la UNED de España ofrece un novedoso Título Propio de Experto Universitario en Educación a Distancia y Formación Virtual. También empiezan a integrarse, como parte de ese esquema, los proyectos de vinculación universidad-empresa y los llamados perfiles de salida "a la medida".

La nueva ética universitaria. Las modificaciones en la forma en que los valores son identificados y compartidos en la sociedad impactan, necesariamente, la forma en que esas sociedades perciben el rol de los universitarios en ella. Recientemente, algunos cambios han tenido efectos asombrosos. En algunas áreas de actividad, la preocupación por los aspectos morales es mucho más evidente, como por ejemplo en la difusión y la adhesión al nuevo paradigma socio-ético en relación con la conservación y el reciclaje, aunque algunas veces sea solo una promesa vacía y sin voluntad de compromiso. Lo indiscutible es que en la universidad se refleja en modelos de conducta que afectan el uso de recursos, el tratamiento de desechos y, dentro de estos, en especial, el papel. En contraposición, se habla de la universidad virtual, menos consumista y más fácil de acceder (con menos consumo de energía).

En forma similar se ha desarrollado la que se ha llegado a identificar como cultura de la calidad: Actualmente, en el lenguaje cotidiano en el claustro se habla de "vocación de calidad", "control de calidad", "evaluación y acreditación". Característicamente, en la UNED existe un mandato expreso del Consejo Universitario, al mismo tiempo que, en las unidades de la DPM, prevalece la decisión interna de procurar un modelo de calidad digital para toda la producción.

Expansión de la oferta de programas universitarios desde y hacia el exterior. Las características del mercado para los programas y cursos basados en tecnologías innovadoras se han modificado notablemente en los tiempos recientes. El tamaño del mercado universitario en general y de los mercados específicos de algunos cursos o materias se ha multiplicado explosivamente. En los años que corren, se oye cada vez con más frecuencia sobre la producción orientada a un marco geográfico mayor, como Centroamérica; o sobre cursos o programas regionales, o con el énfasis orientado a determinado país. De forma inversa, la invasión de multiplicidad de organizaciones del exterior que ofrecen variadas alternativas de educación, con grandes facilidades, es notoria.

Un par de lustros atrás, la oferta de educación universitaria a distancia de la UNED se encontraba prácticamente sola en el país. Hoy son numerosas las opciones ofrecidas por otras universidades 
y sistemas nacionales, incluso los de más rancia vocación presencial, que abren sus servicios y ofrecen alternativas si no abiertas, por lo menos mixtas, combinando con el aula diversidad de plataformas y redes para la comunicación remota. En esta época, la UNED enfrenta competencia directa en su propio mercado, con las tecnologías y las herramientas que le parecían propias.

\section{La naturaleza mutable de la innovación"}

Hace apenas unos cuatro años, en congresos y en otras actividades académicas sobre el uso de tecnologías en educación, planteábamos que la forma más novedosa, provocativa y con mayor potencial que podríamos adoptar, para conducir la relación docente y la gestión del sistema de estudiantes en una universidad a distancia, era mediante un conjunto importante de cajeros automáticos distribuido por todo el país. Proponíamos sustituir los centros académicos existentes por un servicio automático de conexión remota con la Universidad, lo que se conoce - del ingléscomo una red de ATM.

Estas máquinas serían extensiones de todos y cada uno de los servicios provistos por la Universidad a los estudiantes. El estudiante entraría a todos los sistemas utilizando los recursos normales del cajero para administrar bases de datos en forma remota. El sistema contaría con previsiones para la identificación correcta de los usuarios y para garantizar la seguridad de las operaciones. Las aplicaciones tecnológicas de punta en ese momento, permitían anhelar esta fantasía.

Pero en menos de dos años, en el Congreso Internacional de Tecnología y Educación a Distancia organizado por la UNED en San José en noviembre de 2006, alcanzábamos la extraordinaria conclusión de que la alternativa de utilizar cajeros automáticos para la entrega de la educación a distancia, empezaba a lucir obsoleta... ¡sin siquiera haber intentado llevarla a la práctica! Propusimos entonces algo que apenas se avizoraba en el horizonte. Una visión transformadora, basada en las tendencias principales que se percibían, que tendía a privilegiar el desarrollo y el potencial de dispositivos individuales personalizados. El creciente uso, estimulado tanto por moda como por precio, de aparatos pequeños, de operación inalámbrica y con bajo consumo de energía, sugería que en un corto plazo su presencia será universal. ¡No nos imaginábamos cuán pronto!

Con respecto al hardware, proponíamos que el estudiante dispusiera de un equipo portátil combinado que garantizara el funcionamiento en diferentes lugares, y que fuera potente para facilitar el correcto funcionamiento de aplicaciones multimedia, reproducción de videos y comunicación en tiempo real, entre otros recursos y que permitiera comunicación con diferentes tipos de redes. La conexión $W i F i$ de banda ancha le serviría para navegar en Internet por medio de redes inalámbricas en centros comerciales, bancos, centros universitarios y otros lugares públicos. Esta conexión también permitiría a los estudiantes enlazarse entre sí mientras se encontraran en tutorías o centros de estudio. Por último, se sugerían enlaces conmutados y con redes más poderosas (WiMAX).

El equipo ideal, decíamos, constaría de una computadora portátil estilo tablet y un teléfono celular tipo smartphone. Esta computadora es, primero, una computadora personal normal con

\footnotetext{
"Esta sección se ha escrito con la colaboración de Marco Antonio Sánchez.
} 
todo su potencial y facilidades. Segundo, su pantalla es táctil y funciona como "una hoja de papel" en donde se puede escribir directamente, como en la pantalla de una agenda electrónica, lo que da la posibilidad de utilizarla para tomar notas, escribir, dibujar, diseñar, entre otras funciones. Como característica especial la computadora dispondría de un sistema de sonido y cámara (webcam) para poder realizar "video conferencias" con compañeros, tutores y otros, utilizando Internet. Asimismo, contaría con un dispositivo lector de huellas dactilares para que pudiera realizar tareas que exigieran garantías de seguridad.

El Smartphone es un teléfono celular con servicio de mensajería SMS -para mensajes de texto-, MMS - para mensajes multimedia- y navegación por Internet. Este teléfono se conecta con la computadora por medio de un sistema inalámbrico bluetooth para realizar la transferencia de datos entre ambos dispositivos y para establecer la conexión a Internet de la computadora. El bluetooth le permitiría a estos dos dispositivos enlazarse remotamente con otros: cámaras fotográficas, cámaras de video, audífonos inalámbricos, otras computadoras, etc. Asimismo, un componente del teléfono sería una cámara fotográfica digital de al menos 2 megapixeles de resolución (calidad de la imagen) para brindarle apoyo al estudiante en la elaboración de sus materiales. El smartphone contaría con un sistema de agenda para mantener actualizado al estudiante con respecto a fechas de actividades mediante sincronización con la computadora. También, tendría capacidad para reproducir archivos de sonido y de video para poder escuchar y ver las entregas de podcasts (archivo MP3 -audio-) y vodcasts (archivo MPeg4 -video-). En relación con el software, si bien es cierto que ambos dispositivos funcionan de una forma integrada, debe hacerse la diferencia entre las aplicaciones que se ejecutan en la tablet y las que se ejecutan en el smartphone. Este tema, aunque importante, no se detalla aquí, dado que lo esencial de la "novedad" de la propuesta queda suficientemente explicado.

En la actualidad, todos tendemos a estar "conectados" telefónicamente y por medio de Internet, y en educación a distancia, en medio de tan intricados circuitos de comunicación, la seguridad tiene una razón particular de ser. Por un lado, existe la necesidad de mantener privada la información del estudiante: que nadie pueda utilizar su computadora y revisar la información que tenga ahí; problema que se soluciona con los lectores de huellas dactilares que se incorporan en las computadoras. Por otra parte, la necesidad de garantizar, al emitir certificaciones o al aplicar evaluaciones, que quien se encuentra actuando a distancia, es el estudiante que tiene el derecho a hacerlo. En este caso, no existe un dispositivo electrónico que permita garantizar la seguridad absoluta, por lo que, lo que debe cambiar-seguramente- es el sistema de evaluación.

Hasta ahora se han presentado las principales innovaciones tecnológicas en manos de los estudiantes. Exagerando la figura, podría hablarse del extremo receptor de la comunicación. Pero lo cierto es que, como contrapartida, en la sede de la Universidad deben de adoptarse modificaciones acorde con la misma revolución tecnológica.

Por ejemplo, para acortar distancia y acercar a las personas se necesita una conexión en red "ambiciosa" y eficiente. La Universidad deberá transmitir una gran cantidad de datos a estudiantes y a tutores, y entre diversas instancias académicas y administrativas. Para lograr este cometido se requiere que los estudiantes cuenten con banda ancha de conexión a Internet. Esta 
conexión de alta velocidad debe ser accesible por medio de una red convencional (con cables), o utilizando conexiones inalámbricas, como las WiFi y WiMAX ya comentadas.

La Universidad debe contar con una plataforma para el aprendizaje en línea potente y robusta. También debe ser muy flexible para poder adaptar interfases y otras estructuras, así como desarrollar metáforas comunicacionales que multipliquen el valor informativo y formativo de los materiales. La página WWW de la universidad debe ser un símbolo y un estandarte fácil de identificar, inspirador, provocativo, que invite a navegar y a quedarse. El proceso de enseñanza y aprendizaje exitoso comienza desde que se puede propiciar este amor a primera vista.

La Universidad debe fortalecer sus sistemas de producción y, en especial, de posproducción. Esto es vital en cuanto se refiere a producción radial y televisiva, así como a las videoconferencias. En estos campos, eventualmente, pero en un plazo muy corto, todos los productos deberían ponerse a disposición de los públicos utilizando cuentas bajo pedido en Internet. Más atrás en la cadena de producción, hay que ampliar, renovar y mejorar los estudios de grabación y los otros espacios de producción. En correspondencia con la expectativa tanto la producción como la transmisión tenderán a ser en formatos digitales; en un futuro inmediato todos los equipos para grabación, edición y postproducción deberán corresponder a esa tecnología.

En los procesos de producción, la unidad se logrará adhiriéndose, crítica pero constructivamente, a tendencias filosóficas que fortalecen el concepto de unidad en el diseño, así como el papel central de las metáforas comunicacionales como parte de un desarrollo consciente de una teoría de la imagen aplicada. El diseño universal, el diseño instruccional, el diseño emocional y el conocimiento como diseño, son modos del pensamiento que irán creando espacios para lograr un mejor uso de los recursos y un mayor compromiso de cátedras, profesores y autores de materiales didácticos.

Aunque puede parecer sencillo -pero no es en absoluto trivial-, habría que fortalecer el uso de las versiones actualizadas de software como el power point y el flash, así como la adopción de algún hardware (ie video toaster) con propósitos similares, para entrenar a los profesores y tutores en el desarrollo de sus propios materiales multimediales para el uso en conferencias y otras actividades.

Hay que aprovechar más y mejor la forma en que las aplicaciones basadas en la WWW pueden ayudar en el aprendizaje. Se debe tener claro que todas ellas se desarrollan para ejecutarlas en el servidor de la Universidad (o en un servicio de outsourcing, pero dedicado) para dar un apoyo determinado a los estudiantes que ingresen a ese servidor. Todas las aplicaciones de este tipo, los foros, las bitácoras (blogs), los wikis y los chats, deben obedecer a una estrategia "académica" del diseño: que puedan ser objeto del diseño curricular, y que puedan ser cubiertas por metáforas pedagógicas ricas y convincentes.

El administrar foros y bitácoras exigirá que se incluya, dentro de la carga de trabajo de los tutores, el tiempo conveniente para brindar la atención correcta. Del mismo modo, se hace necesario darles el entrenamiento para hacer más efectivo su trabajo.

Hay que propiciar que los estudiantes adquieran un servicio de correo electrónico. La Universidad puede desarrollar un sistema interno, pero es más fácil utilizar alguna de las 
aplicaciones WWW más conocidas, como: Yahoo o Hotmail. La estrella, sin embargo, es Google con su sistema de correo (gmail) que ofrece hasta 6,6 GB de espacio (a principios de 2008). Esta aplicación ha evolucionado y permite hoy que el usuario defina su propia página de acceso, en la cual se depositan automáticamente todas las novedades en las áreas de su interés, y ofrece conjuntamente el servicio de mensajería instantánea de texto y de voz. También facilita el acceso directo a una voluminosa videoteca, creada por los usuarios de Google.

Por otro lado, deberán valorarse continuamente los nuevos desarrollos de aplicaciones a los contextos pedagógicos. Actualmente, hay un sostenido avance de las plataformas de aprendizaje en línea y se multiplican las facilidades integradas con programas completos ofrecidos por prestigiosas universidades, con expectativas exclusivamente de difusión y servicio. La llamada Web 2.0, que más bien parece una caracterización de la nueva conducta participativa, creativa e independiente, promovida por los desarrollos en los sistemas de comunicación, y también las nuevas facilidades en potencial de diseño, incluyendo $3 D$ y Second Life, sugieren que el universo al alcance de los medios educativos tiende a ampliarse exponencialmente.

Algunos ejemplos de proyectos concretos realizados por otras universidades ilustran este potencial. A continuación se presenta un ejemplo de la Universidad de Carleton, Canadá, y otro de la Universidad Nacional Abierta de Corea.

Experiencia en la Universidad de Carleton, Ottawa, Canadá. El incremento en el uso de los reproductores MP3 en Norteamérica ha permitido que las universidades distribuyan material adicional para algunas clases en ese formato. Desde hace algún tiempo algunas universidades han puesto a disposición de sus estudiantes, para que los descarguen a sus computadoras y reproductores, archivos de audio en Internet. Pero, la experiencia de la Universidad de Carleton es trascendente, pues han colocado todo un curso de química en vídeo para los reproductores personales. El profesor a cargo de este plan piloto afirma" que no pretenden eliminar las clases presenciales, sino administrar el tiempo que tradicionalmente se utilizaba en ellas para dar una atención y resolución de dudas más personalizada, pues los estudiantes van a ver los materiales con anterioridad y llevarán sus consultas a clase.

Una limitante de esta iniciativa es que no es posible utilizar esta modalidad en todos los cursos, dado que la mayoría usa materiales didácticos con derechos de propiedad que no le pertenecen a la universidad ni a los profesores.

Experiencia en la Universidad Nacional Abierta de Corea. El profesor Bowon Kim, Director del Instituto de Educación a Distancia de la Universidad Nacional Abierta de Corea del Sur, Seúl, en una conferencia pronunciada hace un par de años, informó que en su universidad se ofrecen, en numerosas materias, conferencias por radio utilizando todos los formatos. Pero lo más llamativo es que todas las conferencias se pueden bajar en formato $M P 3$ del sistema $L O D$ (bajo pedido) de Internet. Muchas de las conferencias se ofrecen también por TV, utilizando una estación convencional y en Internet $L O D$. Posiblemente, esta sea una solución realmente efectiva al problema de ofrecer clases y materiales didácticos a más de un centenar de miles de estudiantes.

" Patrick Lyons. Comunicación personal, enero de 2007. 
El futuro es un vacío que, en esencia, solo podemos llenar recurriendo a nuestra imaginación.

George Shackle

Estos ejemplos sugieren algunas de las características que adoptarán las tecnologías de comunicación en la educación a distancia: se usarán medios y equipos individuales, con los que se accederá a una oferta muy amplia y diversa, producida y transmitida digitalmente por redes muy potentes y con mucha redundancia. Los equipos del futuro serán más fáciles de portar, muy legibles y multifuncionales. Ya se empiezan a ofrecer en el mercado equipos que integran las facilidades que aquí se atribuyen, por aparte, a la tablet $P C$ y al smartphone. Estos cambios en el hardware van a ser importantes, aunque no la clave de la innovación, por ahora.

Muy pronto, los estudiantes podrán elaborar sus trabajos sobre los materiales en sitio o en forma asincrónica; por ejemplo, utilizarán dispositivos de almacenamiento de alta densidad en los que copiarán los materiales que necesitan para su aprendizaje y las actualizaciones de los foros, para poder repasarlos posteriormente. Podrán hacer más trabajo compartido y se contará con facilidades de mayor interactividad. En los próximos años, proliferarán las bitácoras $(b \log s)$, los foros y los wikis, y se incrementará el uso que harán de ellos los estudiantes en sus actividades. Frente a eso, la Universidad tendrá que responder con una plataforma de diseño muy poderosa, capaz de realizar producción de excepcional calidad, muy accesible por Internet y con numerosas facilidades al servicio de los estudiantes.

El futuro, en estos términos, ya nos ha alcanzado. El reto es imaginar la sociedad que queremos y asumir su construcción con el apoyo de la innovación recurrente.

\section{REFERENCIAS}

Fabré Redondo, B. (2006). Las nuevas tecnologías de la información en la educación y la medicina. Recuperado el 19 de setiembre de 2006, de http://cis.sld.cu/E/monografias/teleeducacion.PDF

González, W. J. (editor). (2007). Las ciencias del diseño: Racionalidad limitada, predicción y prescripción. La Coruña: Netbiblo, S.L.

Henry, J.; Walker, D. (1991). Managing innovation. Londres: Sage-The Open University. ${ }^{1}$

Heskett, J. (2002). Design. A very short introduction. New York: Oxford University Press. [Heskett J. (2005). El diseño en la vida cotidiana. Barcelona: Gustavo Gili.]

Johnson, S. Here comes everybody, Discover, July 2006, p.23.

Lidwell, W.; Holden K. and Butler J. (2003), Universal Principles of Design. New York: Rockport Publishers. [Lidwell, W.; Holden, K. y Jill Butler (2003), Principios Universales de Diseño. Barcelona: Blume.]

Lupton, E.; Abbott Miller J. (eds.). (1999). The ABC's of the Bauhaus and design theory. Boston: Princeton Architectural Press. [Lupton E.; Abbott Miller J. (eds.). (1999). El ABC de la Bauhaus y la teoría del diseño. Barcelona: Gustavo Gili.] ${ }^{2}$ 
Moore, G. E. (1965). Cramming more components onto integrated circuits. Electronics, 38(8), 419-65, 114-117.

Muller-Brockman J. (1998). Historia de la comunicación visual. México: Gustavo Gili.

Multimedia. (1998). Diseño de Sara Day Graphic Design, versión de texto de Eugeni Roselli i Miralles, México: Gustavo Gili. ${ }^{3}$

National Academy of Sciences -USA- . (1993). Responsible Science: ensuring the integrity of the research process. Washington: National Academy Press.

Norman, D. A. (2004). Emotional design. New York: Basic Books [Norman, Donald A. (2005). Diseño Emocional. Barcelona: Paidós.]

Pérez Tornero, J. M. (2000). Comunicación y educación en la sociedad de la información. Barcelona: Paidós.

Perkins, D. N. (1985). Conocimiento como diseño. Bogotá: Pontificia Universidad Javeriana.

Rogers, Everett M. y Shoemaker F. (1974). La comunicación de innovaciones, un enfoque transcultural. México: Herrero Hermanos. ${ }^{4}$

Swann, A. (1989). Layout source book. Londres: Quarto Publishing PLC-The Wellfleet Press. ${ }^{5}$

Villafañe, J.; Mínguez, N. (2002). Principios de Teoría General de la Imagen. Madrid: Pirámide.

Cuatro títulos sobre filosofía del diseño:

Flusser, V. (1999). Filosofía del diseño. Madrid: Editorial Síntesis. (Original en inglés The Shape of Things, Reaktion Books.)

Heller, S.; Pomeroy K. (1997). Design literacy. Understanding graphic design. New York: Allworth Press

Holland DK, (Ed). (2001). Design issues. How graphic design informs society. New York: Allworth Press

Swanson, Gunnar. (Ed). (2000). Graphic design and reading, explorations of an uneasy relationship. New York: Allworth Press

Otras fuentes:

Introduction to universal instructional design at the University of Guelph, fotocopia ( s.f.)

Kim, B. La adopción de las tecnologías telemáticas en las universidades abiertas de Asia: el caso de la Universidad Nacional Abierta de Korea del Sur (KNOU). Conferencia presentada en el Congreso Mundial de Educación Superior a Distancia. La megauniversidad, un reto a la imaginación histórica, Bogotá, Colombia, 4-7 Julio 2006. 
UNED (2006). XIII Congreso Internacional de Tecnología y Educación a Distancia - La educación a Distancia ante los desafios de la democratización del conocimiento. San José, Costa Rica:, Universidad Estatal a Distancia.

\section{NOTAS}

1 Jane Henry y David Walker en Managing innovation estudian los factores organizacionales que favorecen las innovaciones y se enfocan en la forma en la administración puede impulsar y generalizar la creatividad. Analizan el uso de instrumentos como el planeamiento estratégico y el liderazgo visionario en esas actividades. No sorprende encontrar que uno de los autores es una sicóloga y el otro un diseñador y arquitecto.

${ }^{2}$ La Bauhaus es el fenómeno más ampliamente conocido, debatido, imitado, diseccionado y difundido del diseño moderno. Pero en las últimas décadas la educación en el diseño ha olvidado unos de sus legados más importantes: la necesidad de reflexionar sobre el diseño de un modo teóricamente introspectivo. Este libro proporciona la oportunidad de revigorizar el diseño gráfico actual revisando las ideas de la Bauhaus y alentando el pensamiento crítico de los medios y los fines del diseño. Analiza los orígenes y el impacto de la Bauhaus en relación con el diseño básico, el diseño gráfico y la tipografía. Mientras que el texto es una incitante búsqueda de los objetivos y los logros de la Bauhaus, el libro en sí mismo es un manifiesto de sus ideales, en una síntesis de concepción editorial, tipografía y artesanía.

${ }^{3}$ De Multimedia (1998) hay una versión similar producida por Gustavo Gili en Barcelona. El original fue producido por Rockport Publishers de New York. Rockport y Allport en inglés, así como Gustavo Gili en castellano, son las editoriales que ostentan el liderazgo en producciones en el campo del diseño gráfico.

${ }^{4}$ La primera edición del libro La comunicación de innovaciones, un enfoque transcultural de Rogers, y Shoemaker (1974) se publicó en 1962 y todavía no se ha superado el método y el rigor con que explican el grado, el ritmo y el compromiso con que se difunden y se adoptan las innovaciones en la sociedad. Los casos de estudio, que mantienen la pertinencia y la actualidad, captan el interés del estudioso.

${ }^{5}$ El Layout Source Book incluye más de 400 ejemplos de notables producciones realizadas por diseñadores, tomadas de libros, revistas, periódicos, desplegables, boletines, tiras cómicas y anuncios. Organizado en cinco grandes periodos, permite acercarse a los estilos de cada época siguiendo el desarrollo de los productos más típicos de cada una de ellas. 